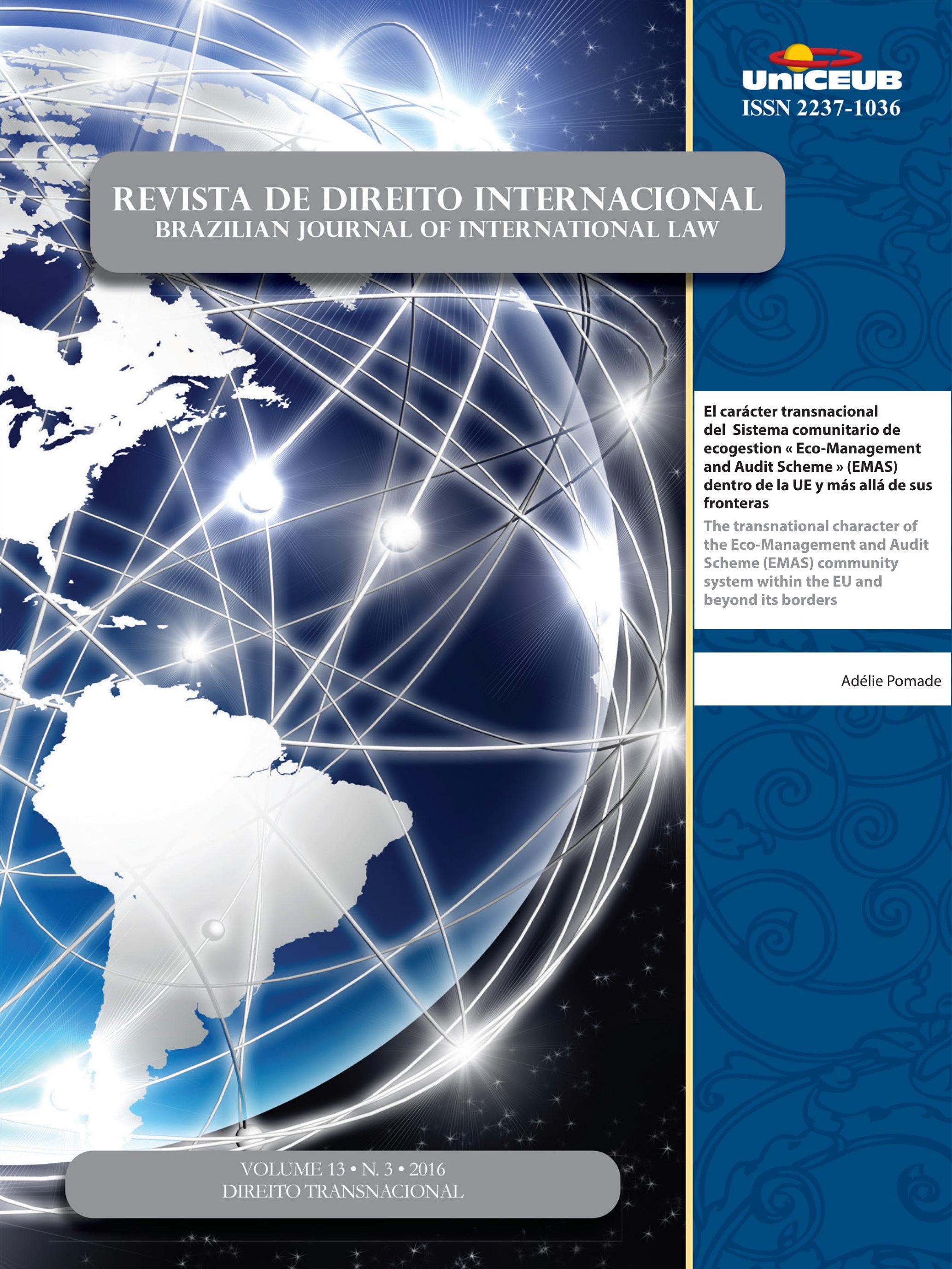


Crônicas da ATUALIdAde do direito internacional .................................................. 2

I. Dossiê Temático: Direito Transnacional .........................................................15

EDITORIAL: O Direito Transnacional - Circulação de normas e relações jurídicas transnacionais .......16 Priscila Pereira de Andrade

A emergênCia do direito transnacional ambiental .............................................18 Priscila Pereira de Andrade

Desafíos y RESPUESTAS TRANSNACIONALES FRENTE A LOS CRÍMENES AMBIENTALES ...............30 Rosmerlin Estupiñan-Silva

DiREITO TRANSNACIONAL E MUdANÇAS CLIMÁTICAS .50 Géraud de Lassus Saint-Geniès

Especies en movimiento: la Convención sobre el Comercio Internacional de Especies Amenazadas de Fauna y Flora Silvestres como espacio de “Encuentro” de discursos, ACTORES Y ESTRATEGIAS EN EL DERECHO AMBIENTAL TRASNACIONAL

María Valeria Berros e Dabel Leandro Franco

El carácter transnacional del Sistema comunitario de ECogestion « Eco-ManageMENT AND Audit SCHEME » (EMAS) DENTRo de LA UE y MÁs ALlÁ DE SUS Fronteras ......72 Adélie Pomade

O CONCEITO DE CONDUTA EMPRESARIAL RESPONSÁVEL À LUZ DOS ORDENAMENTOS JURÍDICOS BRASILEIRO, INTERNACIONAL E TRANSNACIONAL

Gabriel Webber Ziero

ARBITRAGEM NO DIREITO TRIBUTÁRIO INTERNACIONAL E NO DIREITO INTERNACIONAL DOS INVESTIMENTOS: UMA MANIFESTAÇÃO DO DIREITO TRANSNACIONAL

Vivian Daniele Rocha Gabriel 
O DIREITO TRIBUTÁRIO SOB UMA PERSPECTIVA TRANSNACIONAL

Franciele de Simas Estrela Borges

As Características do Direito Transnacional como Metodologia: Análise sob o enfoQue dos Aspectos Processuais da Arbitragem 126

Flávia Foz Mange

O DIREITO TRANSNACIONAL (“GLOBAL LAW") E A CRISE DE PARADIGMA DO ESTADO-CENTRISMO: É POSSÍVEL CONCEBER UMA ORDEM JURÍDICA TRANSNACIONAL? ...................................... 146

Luiza Nogueira Barbosa e Valesca Raizer Borges Moschen

TransPorte AÉREO E DIREITO TRANSNACIONAL: DA CONVERGÊNCIA À UNIFORMIDADE 160 Mickael R. Viglino

Outros Artigos. 175

O Fundo Monetário Internacional e a proteção dos direitos humanos: uma análise DO PROGRAMA DE CRESCIMENTO E REDUÇÃO DA POBREZA NO HAITI 177

Pablo Henrique Hubner de Lanna Costa e Carlos Alberto Simões de Tomaz

Um estranho no ninho? Padrões privados no Acordo de Barreiras Técnicas ao CoMÉRCIO DA OMC 192

Michelle Ratton Sanchez Badin e Marina Yoshimi Takitani

Os benefícios tributários do programa Inovar-Auto e os princípios da Nação Mais Favorecida e do Tratamento Nacional: uma análise dos argumentos dos Painéis atualmente em Curso contra o Brasil no Órgão de SoluÇão de Controvérsias da OMC . 211 Eric Moraes Castro e Silva

A ERA DA HUMANIDADE: REFLEXões PARA A HISTÓRIA DO DIREITO INTERNACIONAL 236 Henrique Weil Afonso

Precedentes vinculantes nos Estados Unidos da América e no direito brasileiro: Um ESTUDO COMPARADO 264

Patrícia Perrone Campos Mello 
IL DIRITTO AMBIENTALE SECONDO L'OTTICA DEL DIRITTO COSTITUZIONALE POSITIVO E LA RESPONSABILITÀ PER DANNI ALL'AMBIENTE NEL DIRITTO COMUNITARIO: LO STATO DELL'ARTE DEL DIRITTO AMBIENTALE COSTITUZIONALE E COMUNITARIO 287

Elcio Nacur Rezende

DA DESCONSIDERAÇÃo DA PERSONALIDADE JURÍDICA NAS RELAÇÕES CONSUMEIRISTAS BRASILEIRAS: ANÁLISE À LUZ DAS TEORIAS CLÁSSICAS

Daniel Amin Ferraz e Marcus Vinicius Silveira de Sá

ANALYSIS OF ADVANTAGES AND DISADVANTAGES OF FORUMS PRESCRIBED UNDER THE UNCLOS AND STATE PRACTICE: THE WAY AHEAD FOR INDIA ......................................................319

Vinai Kumar Singh

Do governo POR LEIS À governanÇA POR NúMERos: breve anÁlise do Trade in SERVICE AgreEMENT (TISA) ...............................................................................338 Jânia Maria Lopes Saldanha, Rafaela da Cruz Mello e Têmis Limberger

As DIRETIVAS EUROPEIAS COMO NORMA REGULADORA DO DIREITO ADMINISTRATIVO GLOBAL ..356 Alice Rocha da Silva e Ruth Maria Pereira dos Santos

O desenVolvimento da POlítica AGRícola COMUM dA UNião EUROPEIA 375 Tatiana de A. F. R. Cardoso Squeff

A imunidade de Jurisdição das organizaÇões internacionais FaCE AO Direito de aCESSO À JUSTIÇA 391

Fernanda Araújo Kallás e Caetano

O DIREITO INTERNACIONAL ENTRE O DEVER ÉTICO E A AÇÃo POLÍ́TICA: OS FUNDAMENTOS DE UM DEVER DE COOPERAÇÃO INTERNACIONAL NA FILOSOFIA POLÍTICA DE IMMANUEL KANT .405 Ademar Junior Pozzatti

EXTENSÃo E FRAGMENTAÇÃo NO CONTEXTO DA JURISDIÇÃO PENAL INTERNACIONAL .423 Marcus Vinícius Xavier de Oliveira

A DEFINiÇÃo JURÍdiCA DA "COMUNIDADE" .444 Nitish Monebhurrun, Michelle Lucas Cardoso Balbino, Fernanda Castelo Branco Araujo, Othon Pantoja, Míara Bogo Bruno e Cândida Dettenborn Nóbrega 
Comparative Study on Chinese Local Legislation of Science and Technology ProGRESS

LI Xiaoming e LI Yihan

O CONTROLE PENAL DO TRÁFICO DE PESSOAS: CONSTRUÇÃO JURÍDICA, INTERAÇÕES ORGANIZACIONAIS E COOPERAÇÃO INTERNACIONAL

Bruno Amaral Machado e Priscilla Brito Silva Vieira

Desativismo judicial: a extradição Battisti no Supremo Tribunal Federal .505 Francisco Rezek e Israel Paulino

A decisão norte-americana do Caso Myriad: novos paradigmas para a Proteção patenTÁRIA DO CÓDIGO GENÉTICO HUMANO E BIOTECNOLOGIA 514 José Carlos Vaz e Dias e Clarisse De La Cerda 


\section{El carácter transnacional del Sistema comunitario de ecogestion « Eco- Management and Audit Scheme " (EMAS) dentro de la UE y más allá de sus fronteras*}

\author{
The transnational character of the Eco- \\ Management and Audit Scheme (EMAS) \\ community system within the EU and \\ beyond its borders
}

Adélie Pomade ${ }^{* *}$

\section{Resumen}

EMAS es un instrumento de gestión desarrollado por la Comisión Europea para las empresas y otras organizaciones cuyo objectivo es evaluar, informar y mejorar su redimiento medioambiental. Abarca todos los sectores económicos y de servicios y es aplicable en todo el mundo. Actualmente, se registran más de 4500 organizaciones y 8.150 sitios. Se encuentran muchas empresas multinacionales y pequeñas empresas, así como las autoridades públicas. Esto es posible gracias a la flexibilidad del instrumento y su adaptabilidad. EMAS se convierte en un instrumento transnacional. En Europa, varios casos se podrá tener en cuenta, incluyendo el Parc Industriel de la Plain de l'Ain en Francia. Internacionalmente, EMAS también sedujo y su aplicación se observa por ejemplo en Trinidad y Tobago. Aquella aplicación «transnacionalizada » pone de relieve una aplicación «uniforme» de la norma europea. Esta observación hecha en la práctica se refiere a una reflexión teórica sobre la recepción social de tales normas flexibles, sino también sus eficacia y eficiencia.

Palabras Clave: Flexibilidad, Sistema de gestión, Redimiento medioambiental , Eficiencia de las normas jurídicas

\section{Abstract}

EMAS is a management instrument developed by the European Commission for Companies and others Organizations which objective is to assess, inform and improve their environmental performance. EMAS can be applied in the worldwide. Today, we count more than 4500 organizations and 8.150 sites concerned by the system. It both concerns multinational companies, small societies and public authorities. The flexibility of this instrument makes possible this broad and transnational application. At European and international levels, various case studies can be taken into account: the Parc Industriel de la Plain de l'Ain in France and the case of Trinidad and Tobago. This transnational application highlights uniform application of the
** Docteur en droit Habilité à diriger des recherches (HDR), Chercheur associé au CEDRE, Université Saint-Louis de Bruxelles (USL-B) - Belgique, Chercheur associé à l'UMR CNRS Institut de l'Ouest: droit et Europe (Université Rennes 1)-France. E-mail: adeliepomade@orange.fr
Recebido em 15/01/2017

Aprovado em 18/01/2017 
European legal norm. This contribution will permit to make a theoretical reflexion on the social reception of legal norms, but also on effectiveness and efficacy of such norms.

Keywords: Flexbility, Management system, Environmental performance, Effectiveness of legal norms

El Sistema de Gestión Ambiental y Auditoría en la Unión Europea (EMAS) es una herramienta de gestión voluntaria para las empresas y otras organizaciones que quieren evaluar, mejorar y dar a conocer su rendimiento medioambiental. EMAS se estableció en $1993^{1}$ por una regulación europea y fue revisado en $2001^{2}$. Su ámbito de aplicación se fue extendido más allá de la industria a todos los sectores (servicios públicos y privados). Gracias a este cambio, todas las empresas, independientemente de su tamaño y sector de actividad, y cualquier tipo de organización (gobierno, áreas de negocio...) están implicados. La norma revisada en $2009^{3}$ y entrada en vigor en enero de 2010 ha extendido una vez más el ámbito de aplicación de EMAS. Gracias a este desarrollo, EMAS se aplica a cualquier organización con sede fuera de la Unión Europea (UE). Esto abrió el camino para el reconocimiento internacional del sistema de gestión.

El objetivo de la reglamentación es establecer procedimientos simplificados y eficaces para incitar a las empresas comprometerse en una acción voluntaria. EMAS es un sistema de gestión ambiental voluntaria que permite identificar las empresas y organizaciones que mejoran continuamente sus resultados en materia de medioambiente ${ }^{4}$.

EMAS está basado en el sistema de gestión ambiental ISO 14001 mientras era acompañado de requisitos adicionales. En este sentido, las ambiciones EMAS son superiores a las de la norma ISO 14001, que, sin embargo, constituye una norma de referencia. EMAS da un paso hacia una mayor eficiencia y transparencia medioambiental. El cuadro siguiente pone de relieve las diferencias más notables ${ }^{5}$ :

\begin{tabular}{|c|c|c|}
\hline & ISO 14001 & EMAS \\
\hline Objeto de la & Certificación & Certificación \\
certificación & de la evolución & de la evolución \\
& del sistema & del rendimiento \\
& de gestión & medioambiental \\
& para un mejor & del sistema de \\
& rendimiento & gestión \\
\hline
\end{tabular}

\begin{tabular}{|c|c|c|}
\hline & ISO 14001 & EMAS \\
\hline $\begin{array}{l}\text { Conformidad } \\
\text { reglementaria }\end{array}$ & $\begin{array}{c}\text { Debe llevarse } \\
\text { a cabo en un } \\
\text { plazo razonable }\end{array}$ & $\begin{array}{c}\text { Es obligatorio } \\
\text { en la fecha de la } \\
\text { certificación }\end{array}$ \\
\hline $\begin{array}{c}\text { Mejora del } \\
\text { rendimiento } \\
\text { medioambiental }\end{array}$ & No se requiere & Obligatorio \\
\hline $\begin{array}{l}\text { Constante } \\
\text { mejora }\end{array}$ & $\begin{array}{c}\text { Chequeado } \\
\text { periódicamente } \\
\text { Sin frecuencia } \\
\text { específica }\end{array}$ & $\begin{array}{l}\text { Chequeado } \\
\text { anualmente }\end{array}$ \\
\hline $\begin{array}{c}\text { Análisis } \\
\text { medioambiental } \\
\text { inicial } \\
\end{array}$ & $\begin{array}{l}\text { Analysis poco } \\
\text { amplia }\end{array}$ & $\begin{array}{l}\text { Analysis muy } \\
\text { amplia }\end{array}$ \\
\hline $\begin{array}{l}\text { Participación } \\
\text { del personal }\end{array}$ & No se requiere & Obligatorio \\
\hline $\begin{array}{c}\text { Declaración } \\
\text { medioambiental }\end{array}$ & Optativo & Obligatorio \\
\hline
\end{tabular}

El método general establecido por la normativa europea EMAS permite evaluar el impacto medioambiental de la actividad sobre el sitio y sus alrededores, fijar objetivos y elaborar instrumentos concretos para reducir los aspectos negativos de este impacto. Más allá de los requisitos del EMAS, la originalidad del mecanismo reside en la total transparencia de las medidas para reducir el impacto sobre el medio ambiente ${ }^{6}$ (organización de la actividad, gestión de tareas, resultados de productos) de cara al público y al personal.

EMAS constituye por lo tanto una etiqueta que la empresa o la organización pueden prevalerse con respecto a sus proveedores, interlocutores económicos y sociales, clientes o al público. Es una garantía de credibilidad y seriedad en el componente medioambiental de la responsabilidad social empresarial ${ }^{7}$. Concebido y elaborado en el espacio europeo, EMAS resulta «exportable» fuera de la UE. En consecuencia, EMAS aparece como una palanca de rendimiento medioambiental de las empresas y organizaciones por todo el mundo ${ }^{8}$. Sin embargo, en la teoría y la práctica, se plantean una serie de preguntas ¿Podemos decir que tiene un grado suficiente de transnacionalidad para aplicarse de manera efectiva y eficaz? ¿Se puede implementar fácilmente, independientemente del país elegido y de la legislación vigente? En primer lugar, el contenido del Reglamento europeo de 2009 se explorará para saber si y en qué medida facilita la transnacionalidad del instrumento (I). En segundo lugar, se dedicará al análisis de casos en la 
Unión Europea y fuera de sus fronteras, para evaluar en la práctica la aplicación de EMAS y su rendimiento medioambiental (II).

\section{1. ¿LA TRANSNACIONALIDAD DE EMAS FACILITADO POR EL Reglamento dE 2009?}

Varios elementos contenidos en el Reglamento de 2009 permiten entrever una transnacionalidad facilitada del sistema. En primer lugar, los objetivos establecidos en los Considerandos del Reglamento reflejan claramente una voluntad de hacer cumplir la etiqueta más allá de la UE. El punto $n^{\circ} 9$ señala explícitamente que EMAS debe ser accesible a todas las organizaciones, tanto dentro como fuera de la Comunidad, si su actividad tiene un impacto medioambiental. El punto $\mathrm{n}^{\mathrm{o}} 11$ hace hincapié en que las organizaciones que aplican otros sistemas de gestión medioambiental y que desean pasar al sistema europeo de gestión deben ser capaz de hacerlo tan fácilmente como sea posible. En este sentido, el punto 11 señala que el régimen EMAS debe tenerse en consideración los otros sistemas de gestión medioambiental. Esto es importante porque plantea la hipótesis de una coordinación entre EMAS y los otros sistemas de gestión para permitir la máxima compatibilidad entre los diferentes sistemas y la máxima adhesión al programa europeo. También sienta las bases para reducir al mínimo el riesgo de conflictos entre los reglamentos y el riesgo de desanimar a las organizaciones que desean adoptar EMAS.

Entonces, el Reglamento adopta un sentido amplio de las organizaciones que puedan adoptar EMAS. En efecto, el artículo 2 define el término «organización» como «la compañia, sociedad, firma, empresa, autoridad o institución establecida en la Comunidad o fuera de ella, o parte o combinación de las entidades mencionadas, con o sin personalidad jurídica, pública o privada, que tiene sus propias funciones y su propia administración». Por otra parte, invita a «pequeñas organizaciones» adherirse al sistema:

- Micro, pequeñas y medianas empresas.

- Las autoridades locales que administran bajo de 10 000 habitantes y otras autoridades públicas que emplean a menos de 250 personas y que tienen un presupuesto anual que no exceda de 50 millones de euros, o cuyo balance general anual no excede de 43 millones de euros (administraciones, servicios públicos, los órganos públi- cos consultivos a nivel nacional, regional o local).

- Personas físicas o jurídicas que ejercen, en virtud del derecho nacional, funciones administrativas públicas, en particular tareas, actividades o servicios específicos relacionados con el medio ambiente.

- Las personas físicas o jurídicas que asuman responsabilidades o funciones públicas o preste servicios públicos relacionados con el medio ambiente.

El carácter global de estas definiciones refleja la flexibilidad del instrumento. Esto permite la adhesión de una amplia gama de organizaciones interesadas por todo el mundo. El aspecto atractivo de la norma que se dirige a un público muy vasto hace de este instrumento un "instrumento fácilmente accesible".

Por último, el anexo IV del Reglamento evoca un conjunto de indicadores básicos que la organización debe incluir en sus documentos de información, y de los cuales su rendimiento medioambiental se valora. Estos indicadores abarcan las áreas de eficiencia energética, la producción de desechos, el uso eficiente de los materiales, la biodiversidad, el uso del agua o las emisiones atmosféricas. Sin embargo, EMAS prevé una aplicación flexible de estos indicadores: la organización puede presentar los indicadores que considera más representativo de su actividad. Sólo se debe demostrar en su análisis del medio ambiente que la inclusión de otros indicadores no es relevante. Esto brinda la oportunidad a la organización beneficiarse de una grande capacidad de adaptación del sistema a su entorno?.

A primera vista, el Reglamento es muy técnico y complejo porque contiene requisitos impuestos a las organizaciones. De hecho, está adaptable y flexible ${ }^{10}$. Esta ventaja es vector del rendimiento medioambiental de la empresa, independientemente de su situación geográfica.

En primer lugar, los criterios establecidos para la adhesión al EMAS y establecer el sistema de aplicación general son estrictos y precisos. Esto garantiza un mayor éxito del instrumento, en términos de adhesión y eficiencia, porque enmarcan la acción de la empresa de cerca, dejándolo un poco de libertad para organizarse. Por ejemplo, para obtener la etiqueta ecológica, la organización debe:

- Adoptar una política medioambiental que define los objetivos y principios de acción de la empresa en relación con el medio ambiente. Según el artículo 2 del Reglamen- 
to, esta política es la expresión de las intenciones y de la orientación del rendimiento medioambiental de la organización. Esto implica el respeto de todos los requisitos legales aplicables en materia de medio ambiente y el compromiso con la mejora permanente del rendimiento medioambiental. Esta política establece un marco y prevé el establecimiento de objetivos medioambientales.

- Producir un análisis medioambiental de sus actividades, productos y servicios. El análisis medioambiental consiste en efectuar un examen más amplio de los impactos y los resultados obtenidos en una serie de ámbitos como el agua, el aire, el ruido, los desechos, el consumo de energía, etc.

- Desarrollar un programa medioambiental (artículo 2) que coincide con la descripción de las medidas, responsabilidades y medios adoptados o previstos para lograr los objetivos y metas medioambientales y los plazos para alcanzarlos.

- Preparar una declaración medioambiental (artículo 2), además de la auditoría medioambiental obligatoria, para comunicar su rendimiento medioambiental. Este documento es la originalidad del sistema. Incluye toda la información proporcionada al público y a otras partes interesadas en relación con: la estructura y actividades (bienes y servicios) de la organización; su política medioambiental y su sistema de gestión medioambiental; su impacto medioambiental; su programa medioambiental y sus objetivos medioambientales generales y específicos.

La información requerida debe ser completa, detallada y transparente, pero la redacción del Reglamento deja claramente a la organización la flexibilidad necesaria para lograr este objetivo.

En segundo lugar, el enfoque requiere una dinámica colectiva que asocia estrechamente los empleados a la implementación de EMAS. En efecto, el Reglamento prevé expresamente en el punto $\mathrm{n}^{\circ} 14$ de sus Considerandos que «deben participar los empleados y trabajadores de la organización, ya que ello aumenta la satisfacción en el trabajo y el conocimiento de cuestiones medioambientales, que puede reproducirse dentro y fuera del entorno laboral». Esta participación plural es un factor de efectividad y de éxito del rendimiento medioambiental, porque multiplica los esfuerzos dentro de la organización ${ }^{11}$. Por otra parte, contribuye a la legitimidad de la norma que se considera por todos como necesaria para el desarrollo de la organización ${ }^{12}$.
En tercer lugar, la verificación del cumplimiento de los compromisos de la organización es frecuente y su radiación parece fácil. La auditoría ambiental se lleva a cabo por un inspector certificado que examina el enfoque de la organización ${ }^{13}$. Desde el inicio del proceso de registro de la organización en EMAS, el verificador medioambiental elabora, en consulta con la organización, un programa que garantice la verificación de todos los elementos requeridos para la acceptación y la renovación del registro (artículo 19 del Reglamento). Esto sugiere un proceso de control más adaptado a las necesidades de la organización. Así podemos estimar que esta flexibilidad es la contrapartida de un escrupuloso respeto de sus obligaciones (información, transparencia...). Por lo tanto, el verificador evalua periódicamente la conformidad de la análisis medioambiantal, de la política medioambiental, del sistema de gestión y de los procedimientos de auditoría de las organizaciones, así como su puesta en práctica. Por lo tanto, una organización que brinda ninguna explicación clara y convincente de la falta de cumplimiento de sus obligaciones, que no produce todos los documentos requeridos o se olvide de informar de cualquier cambio realizado en uno de los documentos puede ser eliminada del sistema o ver su registro EMAS suspendido (artículo 15 del Reglamento).

Una lectura cuidadosa del Reglamento de 2009 indica claramente una voluntad de aplicación facilitada de EMAS, lo que refuerza su carácter transnacional como aplicable a la mayor parte de organizaciones por todo el mundo. Queda por ver si, en la práctica, EMAS logra seducir en la UE y fuera de sus fronteras, y si su aplicación permite realmente a las organizaciones a lograr un rendimiento medioambiental.

\section{2. ¿UNA tRANSNACIONALIDAD EFICAZ EN LA UE Y FUERA DE SUS FRONTERAS?}

En 2015, dentro de la UE, más de 5.000 organizaciones de todos los tamaños y en todos los sectores ya están registradas. Varias experiencias positivas pueden ser evocadas en Francia y Alemania ${ }^{14}$, mostrando el potencial de rendimiento transnacional y medioambiental del instrumento dentro de la UE.

En Francia, la Plaine de l'Ain ${ }^{15}$ es el primer parque industrial europeo certificado ISO 14001 y registrado EMAS. Alberga más de 142 empresas en 900 hectáreas 
de las cuales 140 hectáreas están dedicadas a espacios verdes. Se cuenta con EMAS desde 2001. Gracias a sus esfuerzos, se presenta como una experiencia de responsabilidad social de empresa que ha perfectamente logrado el encuentro entre las dimensiones económicas, sociales y medioambientales. Entre las medidas aplicadas, se puede evocar el uso compartido de vehículos privados. También se puede mencionar el tratamiento biológico de los residuos industriales y aguas pluviales contaminadas por hidrocarburos. Por otra parte, en términos de biodiversidad, el parque lleva una política de «tolerancia cero» con respecto a los productos fitosanitarios, los setos están protegidos con el fin de preservar los hábitats naturales y la calidad del aire se controla por medio de una análisis de liquen. Tenemos que constatar que el éxito del instrumento es igual en otros Estados miembros.

El caso alemán refleja los beneficios que offrece EMAS en relación con el endurecido de los requisitos ambientales en materia de energía. La nueva política alemana contenida en la ley federal sobre los servicios de energía ${ }^{16}$, que transpone la Directiva europea sobre eficiencia energética de 2012, introdujo un requisito general de efectuar una auditoría energética cada cuatro años. La regla se aplica a todas las empresas con más de 250 empleados y que logran una facturación de más de 50 millones. Está claro que las organizaciones que se benefician de EMAS tienen una ventaja seria. A diferencia de la norma ISO 14001, EMAS impone que las empresas se respeten obligaciones de auditoría en materia de energía. Esto les permite verificar rapidamente que cumplen jurídicas las normas alemanas. Eso es sin contar que la etiqueta les permite también participar más fácilmente a los mecanismos de compensación y obtener beneficios de la aplicación de la legislación establecida en el país en materia de desechos o en materia de emisión de gases de efecto invernadero ${ }^{17}$. En consecuencia, beneficiarse primero de EMAS permite, según las legislaciónes, de cumplir las condiciones exigadas para la concesión de determindadas ventajas o pasar algunos controles. Esta etiqueta es una fuerza para una empresa extranjera que desea establecerse en un Estado miembro o trabajar en colaboración con de una de sus organizaciones.

También se puede mencionar el caso de la empresa alemana Werner \& Mertz que desde 1986 persigue una política de gestión medioambiental renovada. Werner \& Mertz $^{18}$ está registrada en el EMAS por más de 12 años, pero sigue honrar y aumentar sus compromisos volun- tarios adhiriéndose a la Etiqueta Ecológica Europea, al Blauer Engel ${ }^{19}$, al Cradle to Cradle Gold ${ }^{20}$, al ISO 14001, ISO $5001^{21}$, o al LEED ${ }^{22}$. EMAS suplanta, dirige y controla a todos estos otros compromisos. El interés de este caso de estudio no radica en su enfoque, pero más en el marco de este enfoque: adoptando un comportamiento determinado, la empresa se beneficia de EMAS y se enmarca dentro de la política europea de economía circular. Si seguimos un razonamiento más corto, podemos decir que EMAS se puede integrar en las dinámicas colectivas transnacionales, europeas y internacionales de reciclado y valorización de los desechos. Por ejemplo, la «iniciativa de reciclaje» diseñada y desarrollada por Werner \& Mertz tiene como objetivo recoger y empaquetar productos de Polyéthylène Terephtalate (PET) echados en «bolsas amarillas» del país. Botellas de PET de la marca Frosch contienen actualmente $80 \%$ de los productos reciclados, pero 20 a $40 \%$ provienen de material que fuera recogido en bolsas amarillas. El resto (60-80\%) provienen de botellas recicladas. El objetivo de la empresa es lograr un envase compuesto a 98,5\% de materiales derivados de los 1,5 millones de toneladas anuales de plástico arrojados a las bolsas amarillas. Eventualmente, ella espera incorporar en su proceso de reciclaje materiales que hasta ahora no pueden ser (por ejemplo, tapones de botellas recicladas que están hechos de otro material). El objetivo es de estimular nuevas tecnologías y de convencer a las empresas que no se encuentran en el sector del reciclaje a utilizar su método ${ }^{23}$.

Fuera de la UE, EMAS también atrajo. El ejemplo de Trinidad y Tobago, y más recientemente de Israel y Serbia demostra el carácter transnacional del instrumento.

En 2013, Trinidad y Tobago, el país caribeño situado en el Mar Caribe frente a las costas de Venezuela, fue uno de los primeros países fuera de la UE que aprobó el Reglamento EMAS. El objetivo era ambisioso porque consiste a implementar el sistema de gestión de toda la isla. El reto de este Reglamento en Trinidad y Tobago se encuentra en el desafío que se ha tenido la isla : conciliar el turismo y la preservación del medio ambiente. Por una parte, la isla cuenta con uno de la zona protegida más antigua del hemisferio occidental: el Waterfall Argyle. Esta reserva forestal esta legalmente protegida desde 1776 por el Parlamento británico. Por otra parte, la isla es de 37 billones de dólares a través del turismo, y los turistas son principalmente europeos. El objetivo era hacer Trinidad y Tobago un catalizador, un ejemplo típico para motivar y animar a otras islas o espacios a 
hacer lo mismo. Entre los indicadores básicos, la isla optó por poner el énfasis en la reducción del consumo de energía (electricidad), la reducción de la dispersión de desechos, la conservación de la biodiversidad y el uso racional del agua. Las diferentes organizaciones afectadas han implicado a sus empleados para garantizar una máxima eficiencia a las medidas adoptadas. Llevaron a cabo una campaña de sensibilización a destino de los turistas a través de visitas guiadas o posteres. Estos esfuerzos de información caen en línea con la política de comunicación deseado por la UE: las organizaciones deben ser capaces de demostrar que están comprometidas en un diálogo abierto con las partes públicas y otros interesados, incluidas las comunidades locales y sus clientes sobre el impacto medioambiental de sus actividades, productos y servicios. En la práctica, estas medidas consisten, por ejemplo, en explicar el interés de la fauna y flora locales para el equilibrio de los ecosistemas y el planeta, inculcar gestos por no malgastar el agua, utilizar con moderación la electricidad, recurrir a la recogida selectiva de vidrio y plástico. El punto fuerte de estas acciones reside en el hecho de que las recomendaciones se acompañan de visitas y de ocio. El objectivo es sensibilizar a los turistas del bienestar que se derivan del funcionamiento de los ecosistemas que se encuentran en la isla. El bienestar del publico depende de su comportamiento. Una primera evaluación positiva se puede extraer de esta experiencia. De hecho, la isla reporta una reducción de residuos de $50 \%$ y una disminución de $10 \%$ en el consumo de energía ${ }^{24}$.

Otro ejemplo de la implementación EMAS fuera de la UE es el enfoque realizado conjuntamente por la Agencia Austriaca para el Medio Ambiente, la Agencia Alemana del medio ambiente y la Company Northern Ireland Cooperation Overseas (NICO) para exportar EMAS en Israel ${ }^{25}$. El enfoque se basa en una asociación creada con el Ministerio de Medio Ambiente de Israel en torno a un proyecto de dos años llamado «Apoyo al Ministerio de Medio Ambiente de Israel para que adopte y aplique EMAS en beneficio de la industria y el sector comercial «. El proyecto se inició en junio de 2015 y la reunión de lanzamiento se llevó a cabo en septiembre y generó los primeros resultados satisfactorios. El primer de ellos fue el mantenimiento de un desarrollo sostenible en el territorio israelí. El proyecto consta de tres componentes que incluyen un componente «EMAS » supervisado por la Agencia Austriaca. Su misión es informar al Ministerio israelí de los requi- sitos administrativos y legales que deben cumplir como parte del sistema. Por otra parte, la Agencia debe realizar un balance para poner de relieve los resultados de las organizaciones registradas en el EMAS.

La implantación de EMAS en Serbia es otra realidad. Bajo los auspicios del proyecto europeo «Law enforcement in the field of industrial pollution control, prevention of chemical accident and implementation of the EMAS system $\rangle^{26}$ iniciado en 2012, las autoridades serbias y la UE lleva a cabo una política de promoción de EMAS y alienta a las organizaciones y empresas a unirse. Tres empresas ya están participando en el proceso de registro con el fin de recibir la etiqueta ecológica antes del fin de 2015. Las empresas participantes son Galenika-Fitofarmacija a.d., un fabricante de pesticidas y productos agroalimentarios, Gorenje d.o.o., un fabricante de equipos domésticos eléctricos y TRS Europa d.o.o., uno de los líderes del reciclaje de cartuchos de tóner de la impresora. Las organizaciones que planean participar en el programa recibieron una sesión de capacitación en Marzo 2013 en Austria y en 2014 recibieron asistencia que les permite integrar el sistema de gestión en su actividad.

En conclusión, podemos afirmar que EMAS proporciona un carácter decididamente transnational. Esta conclusión se desprende no sólo de la lectura del Reglamento de 2009, que presenta la forma más completa, pero también en relación con el éxito de la norma en la UE y fuera de sus fronteras. La transnacionalidad emana de la capacidad de la norma adaptarse a los contextos ecológicos, sociales, económicos y jurídicos de las organizaciónes que deseen participar en el programa. También se destaca por la legitimidad lesea reconocida colectivamente por las organizaciones, independientemente de su ubicación geográfica. EMAS trasciende y supera las barreras técnicas que se pueden encontrar cuando sea necesario cumplir con una serie de condiciones. De hecho, se plantea directrices suficientemente precisas pero flexibles no sólo para permitir una implementación adaptada a los destinatarios, eficaz y eficiente sino también para lograr los resultados deseados.

\section{Bibliografía}

1 Reglamento (CEE) no 1836/93 del Consejo, de 29 de junio de 1993, por el que se permite que las empresas del sector industrial se adhieran con carácter voluntario a un sistema comunitario de gestión y auditoría medio- 
ambientales, DOUE 10/07/1993, p. 1.

2 Reglamento (CE) n 761/2001 del Parlamento Europeo y del Consejo, de 19 de marzo de 2001, por el que se permite que las organizaciones se adhieran con carácter voluntario a un sistema comunitario de gestión y auditoría medioambientales (EMAS), DOUE 24/04/2001, p. 1.

3 Reglamento (CE) No 1221/2009 del Parlamento Europeo y del Consejo, de 25 de noviembre de 2009, relativo a la participación voluntaria de organizaciones en un sistema comunitario de gestión y auditoría medioambientales, DOUE 22/12/2009.

4 Commission européen, Décision du 4 mars 2013 établissant le guide de l'utilisateur présentant les étapes nécessaires pour participer à l'EMAS conformément au règlement $(\mathrm{CE}) \mathrm{n}^{\circ}$ 1221/2009 du Parlement européen et du Conseil concernant la participation volontaire des organisations à un système communautaire de management environnemental et d'audit (EMAS), (3013/131/ UE), JOUE du 19 mars 2013.

5 D. Morrow, D. Rondinelli, Adopting Corporate Environmental Management Systems: Motivations and Results of ISO 14001 and EMAS Certification, European Management Journal, 2002, Vol.20, pp. 159-171;

F. Testa et al., EMAS and ISO 14001: the differences in effectively improving environmental performance, Journal of Cleaner Production, 2014, Vol.68, pp. 165-173.

6 T. Reverdy, Management environnemental et dynamique d'apprentissage, Revue française de gestion, 2005, $\mathrm{n}^{\circ}$ 158, pp. 250 et ss.

7 M. Nieto Antolin, Responsabilidad social corporativa : la última inovación en management, Universa Business Review, 2004, pp. 28-39.

8 F. Iraldo et al., Is an environmental management system able to influence environmental and competitive performance? The case of the eco-management and audit scheme (EMAS) in the European union, Journal of Cleaner Production, 2009, Vol. 17, pp. 1444-1452 ; R. Rowland-Jones et al., An evaluation of current environmental management systems as indicators of environmental performance, Management of Environmental Quality: An International Journal, 2005, Vol. 16, pp. 211 - 219 ; Cabinet Paul de Baker et ADEME, L'impact économique et l'efficacité environnementale de la certification ISO 14001 /EMAS des entreprises industrielles, ADEME, Juillet 1999 ; M. Personne, Contribution à la méthodologie d'intégra- tion de l'environnement dans les PME-PMI : Évaluation des performances environnementales, Thèse, ENS Lyon, 1998.

9 M. Pâques, Instruments souples, instruments non contraignants, instruments du marché : une alternative pertinente? Anthémis, 2010.

10 A. Prats. Documentation d'un système de management environnemental. Techniques de l'Ingénieur, Techniques de l'Ingénieur, 2011.

11 V. Biondi et al., Environmental Management Systems and SMEs, Greener Management International, 2000, Vol. 29, pp. 55-69.

12 D. Marcel, Articuler normes volontaires privées et règlementations publiques, Perspective, Cirad, n 11, Août 2011.

13 Este inspector se llama «verificador mediambiental». Puede ser un organismo de evaluación de la conformidad o cualquier persona física o jurídica, así como asociaciones o agrupaciones de tales personas, que hayan obtenido una autorización para llevar a cabo la verificación y la validación con arreglo al presente Reglamento. El verificador medioambiental que lleva a cabo la verificación del sistema de gestión medioambiental y la validación de la declaración medioambiental de una organización (o empresa) esta acreditado o autorizado en el Estado miembro en el que dicha organización (o empresa) solicite la inscripción en el registro.

14 J. Freimann, EMAS Experiences in German Companies: A Survey on Recent Empirical Studies, Eco Management and Audit, 2000, n 7, pp.99-105.

15 Parc industriel Plaine de l'Ain, Déclaration environnementale, 2010, http://www.plainedelain.fr/wp-content/uploads/2013/05/Declaration-Environnementale-2010.pdf

16 Referencia jurídica: Energiedienstleistungsgesetz / EDL-G

17 Consultar: http://ec.europa.eu/environment/ emas/news/index_en.htm

18 Consultar : http://werner-mertz.de/Sustainability/Environmental-management-EMAS/

19 Etiqueta ecológica otorgó por el Gobierno alemán a cerca de 4.000 productos y servicios que cumplan estrictos criterios ambientales.

20 Etiqueta certifica que el producto cumple con los altos requisitos cualitativos y ecológicos de la certifica- 
ción "Cradle to Cradle". C2C distingue cuatro niveles: Basic, Silver, Gold et Platinum.

21 Estándar para todas las organizaciones que tiene como objetivo ayudarles a desarrollar una gestión de la energía metódica para mejorar su eficiencia energética.

22 Programa de la OCDE sobre el desarrollo económico y el empleo local (LEED). Su misión es contribuir a la creación de mejores puestos de trabajo a través de una aplicación más eficaz de las políticas, prácticas innovadoras y estrategias integradas a nivel local.

23 Consultar: http://integrally-sustainable.com/index_en.html
24 A. Cooper, Transitioning from a Fossil-Fuel to a Green Economy: Government Policy for Private Sector Participation in Trinidad and Tobago, European Union Foreign Affairs Journal, 2014, n 1, pp. 60 et ss.

25 Consultar: http://ec.europa.eu/environment/ emas/news/index_en.htm

26 EMAS/IPPC/SEVESO - EuropeAid/131555/C/ SER/RS ; Consultar: file://C:/Users/ad/Downloads/IPPC_14258249.pdf 
Para publicar na Revista de Direito Internacional, acesse o endereço eletrônico www.rdi.uniceub.br ou www.brazilianjournal.org.

Observe as normas de publicação, para facilitar e agilizar o trabalho de edição. 